\title{
Comparison of exhaust emission from the most commonly used aircrafts with implementation LTO cycle to operating conditions
}

The LTO cycle (Landing and Take-off cycle) is a research method used for jet engines certification. The measurements are performed in stationary conditions. With the development of aviation, the LTO test started to be carried out more often, but not for its' original purpose. A new aim was an assessment impact of aircraft movement on environment in airports area. LTO cycle consists of four phases (according to ICAO Annex 16): take-off, climb out, approach and taxi/idle. Every airport has different infrastructure, what affects on time during basic flight operations especially for a taxi phase. To rate exhaust emission from aircrafts during basic fly operations duration time should be adjusted to every single airport. As a research area Poznań-Lawica Airport which is located in Poland, was adopted. Based on parameters calculated specially for this airport it is possible to computed there emission from the mostly used aircraft.

Key words: LTO cycle, toxic exhaust gas emission, airlines fleet, adaptation, operating conditions, LTO adaptation

\section{Introduction}

Air transport is the one of the most rapidly developing branches of transport. The increase in the demand for air transport is caused by the competitiveness of this branch primarily in terms of transport time and safety [1]. The Airbus Global Market Forecast indicates that the air traffic doubles every 15 years [2]. It means that as time passes, more and more aircraft will be flying. The issue of the environmental impact of aircrafts is included in Annex 16 of the Chicago Convention, which applies to countries belonging to the International Civil Aviation Organization (ICAO) [3]. It proposed a universal procedure used to assess the emission of harmful exhaust gases from civil aircraft engines. The LTO (Landing and Take-off cycle) is a research method used for jet engines certification. The measurements are performed in stationary conditions. The individual phases of the test correspond to the generalized time of approach, taxiing, take-off and climb out of the aircraft. For civilian airplanes there are four basic test phases (Fig. 1): start $-100 \%$ Fc max (maximum thrust), climb $-85 \% \mathrm{Fc}$ max, landing $-30 \%$ Fc max, taxiing $-7 \%$ Fc max [4]. The entire standard LTO test takes about 30 minutes.

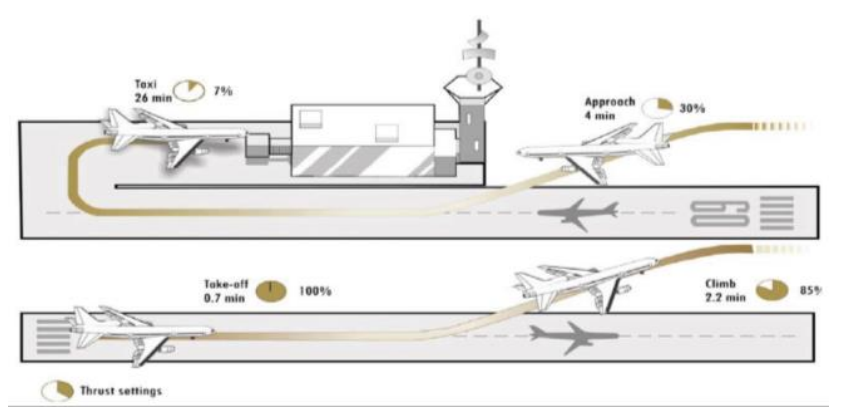

Fig. 1. Landing and Take off cycle [7]

With the development of aviation, the LTO test started to be carried out more often, but not for its' original purpose. A new aim was an assessment impact of aircraft movement on environment in airports area. Each of LTO phases them assigns different thrust and duration time. This methodology is unmatched to conditions prevailing on airports.
Analyzing regulations it should be noted, that duration time do not reflect real conditions during each phase. Every airport has different infrastructure, what affects on time during basic flight operations, especially for taxi phase. Duration time should be adjusted to every single airport. By adjusting the LTO cycle to a given airport, It can be calculated the emission of harmful compounds. In the article, based on the calculated lengths of individual LTO phases tailored to the selected airport, emissions from aircraft most commonly used on the Polish market were calculated.

\section{Research methodology}

As research area Poznań-Ławica Airport was adopted, which is located in Poland. Using the times of the individual phases of the LTO test calculated for the airport in Poznan (Table 1), it was possible to calculate the emission from the most commonly used aircraft. The times of individual LTO phases, for this airport, was calculated in research carried out in article [5], which is focusing on implementation LTO test in flight conditions. The research were carried out using FNPT II MCC simulator.

Table 1. List of regulations and adopted parameters in the LTO cycle [5]

\begin{tabular}{|c|c|c|c|}
\hline \multirow{2}{*}{ Phase } & \multirow{2}{*}{ Thrust } & \multicolumn{2}{|c|}{ Duration time [min] } \\
\cline { 3 - 4 } & & regulations & adopted \\
\hline Take off & $100 \%$ & 0.7 & 0.67 \\
\hline Climb out & $85 \%$ & 2.2 & 1.88 \\
\hline Approach & $30 \%$ & 4 & 5 \\
\hline Taxi/Idle & $7 \%$ & 26 & 9.9 \\
\hline
\end{tabular}

Aircrafts, which are mostly used at Poznań-Ławica Airport are: Boeing B737-400, B737-800 and Airbus A320 and A321. These models were chosen based on fleet of airlines with the largest traffic at Polish market, belong to LCC (Low cost carriers). First aircraft is Boeing 737-400 - the classic model. It is the world's most popular medium-sized passenger airliner. It is produced in the United States. Models 737-400 were produced in the years 1983-2000 and it takes on board from 146 ( 8 in the premium class and 138 in economy) to 188 passengers. Its maximum cruising speed is $912 \mathrm{~km} / \mathrm{h}$ [8]. 
Comparison of exhaust emission from the most commonly used aircrafts with implementation LTO cycle to operating conditions

Table 2. Described of aircrafts chosen to the analysis [8-11, 13-16]

\begin{tabular}{|c|c|c|c|}
\hline Type of Aircraft & $\begin{array}{c}\text { Wing- } \\
\text { span [m] }\end{array}$ & $\begin{array}{c}\text { Maxi- } \\
\text { mum } \\
\text { speed } \\
{[\mathrm{km} / \mathrm{h}]}\end{array}$ & $\begin{array}{c}\text { Number of } \\
\text { passengers } \\
\text { takes on } \\
\text { board }\end{array}$ \\
\hline Boeing 737-400 & 28.88 & 912 & $146-188$ \\
\hline Boeing 737-800 & 28.9 & 840 & $162-189$ \\
\hline Airbus A320 & 34.1 & 871 & up to 180 \\
\hline
\end{tabular}

Next aircraft model is Boeing B737-800. In the basic version, the model 737-800 takes between 162 and 189 passengers on its board. It reaches of $12,496 \mathrm{~m}$, a speed of up to $840 \mathrm{~km} / \mathrm{h}$ and a no-landing range of $5420 \mathrm{~km}$. At the end of February 2016, in Shanghai, Boeing presented the new machine model 737-800, which is the transport version marked as Boeing 737-800 BCF (Boeing Converted Freighter) [12].

Airbus A320 is the most popular model from the entire A320 family. It is the most frequently chosen plane by lowcost airlines, however, its largest operators are traditional, large and medium-sized carriers. This aircraft was produced in both 100 and 200 versions, and the differences were mainly in the length of the range. The first users were Air France and British Airways. The A320 is the first designed aircraft from the entire A318 / A319 / A320 / A321 family [10].

The last aircraft, which is included in analysis is a medium-range passenger plane manufactured by the European Airbus consortium in Toulouse. The Airbus A321 is the largest of the Airbus A320 Family series and the world's largest medium-range passenger plane carrying over 200 passengers. Airbus A321 is a competitor for the American Boeing 737-900 [11].

According to times of the individual phases of the LTO cycle calculated for the Poznań-Lawica Airport it should be noted, that calculated duration time each of LTO phases (Fig. 1) is most different at two phases: approach and taxi/idle. The approach phase is $25 \%$ longer than in regulations and taxi/idle phase is $62 \%$ shorter than in regulations [5]. It can significantly affect to emissions at airport area. Based on parameters calculated specially for this Airport (Table 1) it is possible to computed emission from the mostly used aircraft.

Methodology was concern on computed toxic exhaust fumes emission using the LTO cycle. Emission in LTO cycle is calculated using formula [1]:

$$
\mathrm{EPC}_{\text {pol,mode }}=(\mathrm{TIM} \cdot 60) \cdot \mathrm{FFR} \cdot \mathrm{EF} \cdot \mathrm{NE}
$$

where: $\mathrm{EPCp}_{\mathrm{ol} \text {,mode }}$ - emissions per cycle for a particular mode [g/phase], TIM - Time in Mode [s/phase], FFR - Fuel
Flow Rate [kg/s], EF - Emission Factor [g/kg], NE - Number of engines on aircraft $[-]$.

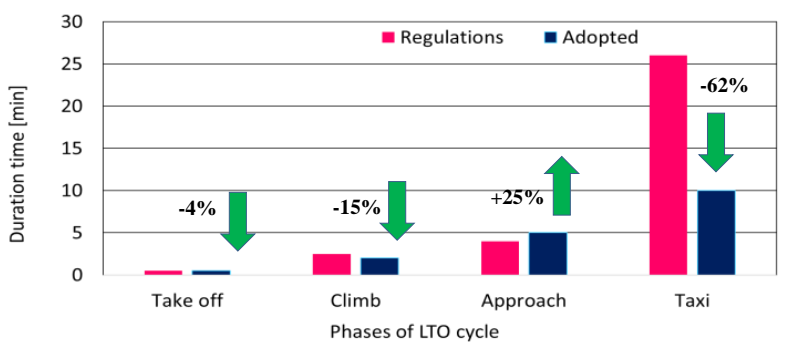

Fig. 6. Differences between LTO time phases in regulations and adopted [based on 5]

Table 3. Emission factors for chosen type of aircrafts [6]

\begin{tabular}{|c|l|c|c|c|c|}
\hline $\begin{array}{c}\text { Type of } \\
\text { aircarft }\end{array}$ & \multicolumn{4}{|c|}{ Emission indicators g/kg } & \multirow{2}{*}{ FF kg/s } \\
\hline \multirow{5}{*}{ B737-400 } & Phase & HC & CO & NO & \\
\cline { 2 - 6 } & Take off & 0.036 & 0.9 & 19.4 & 1.056 \\
\cline { 2 - 6 } & Climb out & 0.047 & 0.9 & 16.7 & 0.878 \\
\cline { 2 - 6 } & Approach & 0.073 & 3.4 & 8.7 & 0.314 \\
\cline { 2 - 6 } & Idle & 1.75 & 30.1 & 4.1 & 0.119 \\
\hline \multirow{5}{*}{ B737-800 } & Take off & 0.02 & 0.25 & 21.79 & 1.213 \\
\cline { 2 - 6 } & Climb out & 0.02 & 0.16 & 17.08 & 0.986 \\
\cline { 2 - 6 } & Approach & 0.05 & 3.07 & 8.93 & 0.331 \\
\cline { 2 - 6 } & Idle & 1.75 & 30.94 & 4.27 & 0.108 \\
\hline & Take off & 0.23 & 0.9 & 24.6 & 1.051 \\
\cline { 2 - 6 } A320 & Climb out & 0.23 & 0.9 & 19.6 & 0.862 \\
\cline { 2 - 6 } & Approach & 0.4 & 2.5 & 8 & 0.291 \\
\cline { 2 - 6 } & Idle & 1.4 & 17.6 & 4 & 0.1011 \\
\hline \multirow{5}{*}{ A321 } & Take off & 0.1 & 0.9 & 33 & 1.051 \\
\cline { 2 - 6 } & Climb out & 0.2 & 0.9 & 26.2 & 0.862 \\
\cline { 2 - 6 } & Approach & 0.5 & 2 & 10.7 & 0.291 \\
\cline { 2 - 5 } & Idle & 3.7 & 19.8 & 4.5 & 0.1011 \\
\hline
\end{tabular}

Based on Aircraft Engine Emissions Databank from 2018 year (Table 3) calculated emission toxic exhaust fumes for following aircrafts: B737-400, B737-800, A320 and A321. Calculations were carried out for emissions in regulations LTO cycle and adopted LTO cycle. The results of calculated emission presented in tables from 4 to 7 .

Table 4. Emission toxic exhaust fumes for Boeing 737-400 in $\mathrm{g} / \mathrm{ph}$ ase

\begin{tabular}{|l|c|c|c|c|c|c|}
\hline \multirow{2}{*}{ Emission } & \multicolumn{3}{|c|}{ Adopted lto } & \multicolumn{3}{c|}{ Regulation lto } \\
\cline { 2 - 7 } & $\mathrm{HC}$ & $\mathrm{CO}$ & $\mathrm{NO}_{\mathrm{x}}$ & $\mathrm{HC}$ & $\mathrm{CO}$ & $\mathrm{NO}_{\mathrm{x}}$ \\
\hline Take off & 3.1 & 76.4 & 1647.1 & 3.2 & 79.8 & 1720.9 \\
\hline Climb out & 9.3 & 178.3 & 3307.9 & 10.9 & 208.6 & 3870.9 \\
\hline Approach & 13.8 & 640.6 & 1639.1 & 11.0 & 512.4 & 1311.3 \\
\hline Idle & 247.4 & 4255.3 & 579.6 & 649.7 & 11175.5 & 1522.2 \\
\hline Sum & 273.5 & 5150.5 & 7173.7 & 674.8 & 11976.4 & 8425.3 \\
\hline
\end{tabular}

Table 4 contains the results of calculations for emissions, toxic exhaust components, performed in $\mathrm{g} / \mathrm{phase}$. Analyzing the results contained in the table, it can be seen that the B737-400 aircraft emits more carbon oxides in regulation LTO test. According to the adopted calculation methodology, it turns out that in the case of adopting the LTO test model based on times adapted for the selected airport, the $\mathrm{CO}$ emission is then about 2 times less. A simi- 
lar situation applies to HC. In the case of nitrogen oxides, the emission in both tests is comparable. Regarding the emissions per test phase, the $\mathrm{CO}$ emissions increase in the idle phase, and $\mathrm{NO}_{\mathrm{x}}$ in the climb out phase.

The largest $\mathrm{NO}_{\mathrm{x}}$ emission in this phase results from the engine's operating conditions at the moment of climbing.

Table 5. Emission toxic exhaust fumes for Boeing 737-800 in g/phase

\begin{tabular}{|l|c|c|c|c|c|c|}
\hline \multirow{2}{*}{ Emission } & \multicolumn{3}{|c|}{ Adopted LTO } & \multicolumn{3}{c|}{ Regulation LTO } \\
\cline { 2 - 7 } & $\mathrm{HC}$ & $\mathrm{CO}$ & $\mathrm{NO}_{\mathrm{x}}$ & $\mathrm{HC}$ & $\mathrm{CO}$ & $\mathrm{NO}_{\mathrm{x}}$ \\
\hline Take off & 2.0 & 24.4 & 2125.1 & 2.0 & 25.5 & 2220.2 \\
\hline Climb out & 4.4 & 35.6 & 3799.3 & 5.2 & 41.6 & 4446.0 \\
\hline Approach & 9.9 & 609.7 & 1773.5 & 7.9 & 487.8 & 1418.8 \\
\hline Idle & 224.5 & 3969.7 & 547.9 & 589.7 & 10425.5 & 1438.8 \\
\hline Sum & 240.9 & 4639.4 & 8245.7 & 604.9 & 10980.4 & 9523.8 \\
\hline
\end{tabular}

Analyzing the data in Table 5, it can be seen that the Boeing 737-800 produces less toxic exhaust fumes compounds than B737-400. As in the previous case, the largest emission concerns on carbon oxides. In the case of the adopted LTO, the CO emission, is much smaller than in regulations test. On the other hand, hydrocarbons are almost three times less. In the case of nitrogen oxides, the emission in both tests is comparable. Regarding the emissions per test phase, the largest emission occurs in phases as in the previously discussed case.

Table 6. Emission toxic exhaust fumes for A320 in g/phase

\begin{tabular}{|l|c|c|c|c|c|c|}
\hline \multirow{2}{*}{ Emission } & \multicolumn{3}{|c|}{ Adopted LTO } & \multicolumn{3}{c|}{ Regulation LTO } \\
\cline { 2 - 7 } & $\mathrm{HC}$ & $\mathrm{CO}$ & $\mathrm{NO}_{\mathrm{x}}$ & $\mathrm{HC}$ & $\mathrm{CO}$ & $\mathrm{NO}_{\mathrm{x}}$ \\
\hline Take off & 19.4 & 76.1 & 2078.7 & 20.3 & 79.5 & 2171.8 \\
\hline Climb out & 44.7 & 175.0 & 3811.6 & 52.3 & 204.8 & 4460.3 \\
\hline Approach & 69.8 & 436.5 & 1396.8 & 55.9 & 349.2 & 1117.4 \\
\hline Idle & 168.1 & 2113.9 & 480.4 & 441.6 & 5551.6 & 1261.7 \\
\hline Sum & 302.2 & 2801.5 & 7767.5 & 570.1 & 6185.1 & 9011.3 \\
\hline
\end{tabular}

Based on the data in Table number 6, it can be seen that the largest emission concerns nitrogen oxides. As in previous cases, the $\mathrm{CO}$ emission is almost 3 times higher in the case of a standard LTO test. On the other hand, hydrocarbons are almost half less when using the LTO test developed for the selected airport. Regarding the emissions per test phase, the largest emission for Airbus A320 occurs in the same phases as in the previously discussed case.

Table 7. Emission toxic exhaust fumes for A321 in g/phase

\begin{tabular}{|l|c|c|c|c|c|c|}
\hline \multirow{2}{*}{ Emission } & \multicolumn{3}{|c|}{ Adopted LTO } & \multicolumn{3}{c|}{ Regulation LTO } \\
\cline { 2 - 7 } & $\mathrm{HC}$ & $\mathrm{CO}$ & $\mathrm{NO}_{\mathrm{x}}$ & $\mathrm{HC}$ & $\mathrm{CO}$ & $\mathrm{NO}_{\mathrm{x}}$ \\
\hline Take off & 8.5 & 76.1 & 2788.5 & 8.8 & 79.5 & 2913.4 \\
\hline Climb out & 38.9 & 175.0 & 5095.0 & 45.5 & 204.8 & 5962.3 \\
\hline Approach & 87.3 & 349.2 & 1868.2 & 69.8 & 279.4 & 1494.6 \\
\hline Idle & 444.4 & 2378.1 & 540.5 & 1167.1 & 6245.6 & 1419.4 \\
\hline Sum & 579.0 & 2978.4 & 10292.3 & 1291.3 & 6809.2 & 11789.7 \\
\hline
\end{tabular}

Table 7 contains emission values of toxic components of the Airbus A321 fumes. As in the previous model, this plane emits the most nitrogen oxides. This model produces almost twice as many hydrocarbons than the Airbus A320.

\section{Analysis and research result}

\subsection{Analysis for Boeing B73-400 and B737-800}

The results of the calculations carried out are presented in the form of diagrams to show the individual dependencies. The sub-section presents and discusses the results for the B737-400 and B737-800. The analysis can be related to approximate course of changes in emission factors: EICO, EIHC, $\mathrm{EINO}_{\mathrm{x}}$, as a function of the Thrust (Fig. 7).

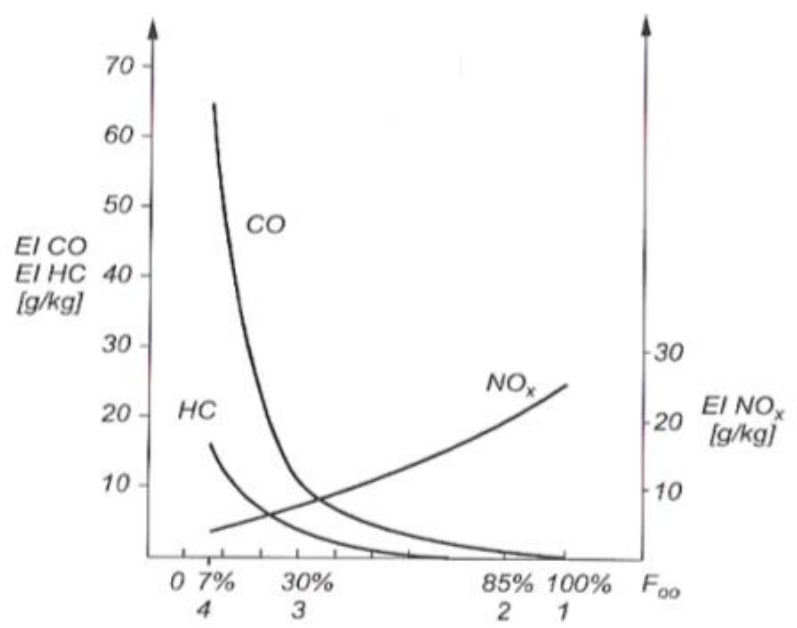

Fig. 7. Approximate course of changes in emission factors: EICO, EIHC, EINO $_{x}$, as a function of the Thrust [5]

It can be seen, that emission factor for $\mathrm{CO}$ is much bigger in taxi and approach phase, than in take-off and climb out phase. The reason of this, is incomplete combustion and local oxygen deficiency. This conditions occur in taxi and approach phase. Which means that $\mathrm{CO}$ emission should be bigger in taxi and approach phase in both cases - adapted LTO and regulations LTO. The same situations consider $\mathrm{HC}$ emission but hydrocarbons emission factor is much smaller than $\mathrm{CO}$ factor. Conditions, which cause big $\mathrm{HC}$ emission are incomplete fuel burn. The opposite situations are in $\mathrm{NO}_{\mathrm{x}}$ cases. $\mathrm{NO}_{\mathrm{x}}$ emission should be bigger in Takeoff and climb out phase and small in taxi and approach phase. This situation is causes by high temperature and pressure in aircraft engine, which occur in take-off and climb out phase, because it's necessary to use the maximum $-100 \%$ and almost maximum $-85 \%$ Thrust in this phases. The analysis should confirm these stated and also demonstrate the differences in results, received in calculation carried out in regulations and adopted LTO cycle.

Figure 8 shows the emission of hydrocarbons in $\mathrm{g} / \mathrm{ph}$ ase in individual phases of the LTO test with the comparison of the emission volumes obtained in LTO regulations and in the LTO adopted for B737-400 and B737-800 for PoznańŁawica Airport. It can be seen, that there is a significant difference in the idle phase for LTO adopted results, HC emission is almost 3 times lower than in the case of standard LTO. It can also be seen that the largest emission of hydrocarbons falls on the Idle phase, which is caused by the longest duration of this phase and the conditions prevailing in the engine. The emission of carbon oxides is much higher than in the case of $\mathrm{HC}$ emissions. The biggest difference in emissions can be seen in the Idle phase. 


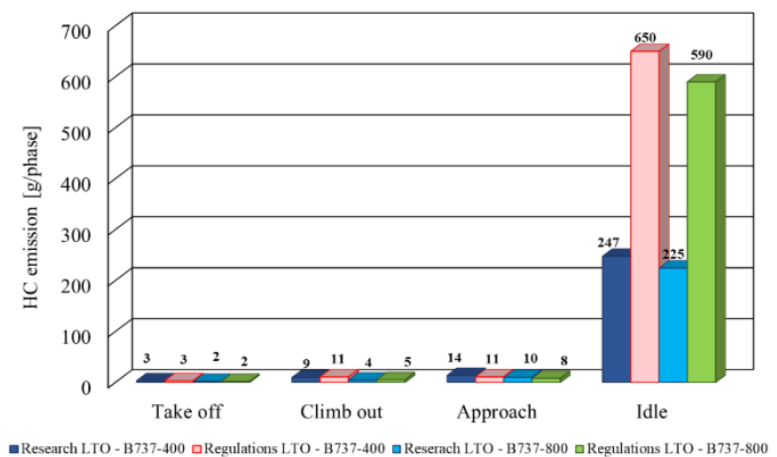

Fig. 8. HC emission for B737-400 and B737-800 in regulations and research LTO cycle in each phase

In the case of regulations LTO, this emission is almost 3 times higher than in the case of LTO referees. A large emission of carbon monoxide also occurs in the Approach phase. In all phase of the test the $\mathrm{CO}$ emission is higher for the B737-400 model. Another chart created is CO emissions in $\mathrm{g} / \mathrm{phase}$, as previously for the two Boeing aircraft models, presented for each phase of both versions of the LTO cycle (Fig. 9).

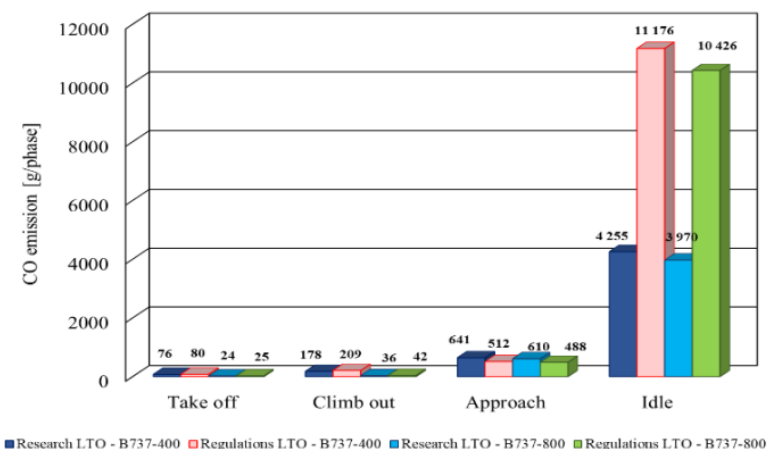

Fig. 9. CO emission for B737-400 and B737-800 in regulations and adopted LTO cycle in each phase

Figure 10 performed the $\mathrm{NO}_{\mathrm{x}}$ emission for the aircraft models in question. The emission of nitrogen oxides differs significantly from other toxic exhaust components. The largest emission is in the climb out phase, because in this phase almost entire engine power is used, and $\mathrm{NO}_{\mathrm{x}}$ are formed at very high temperature conditions.

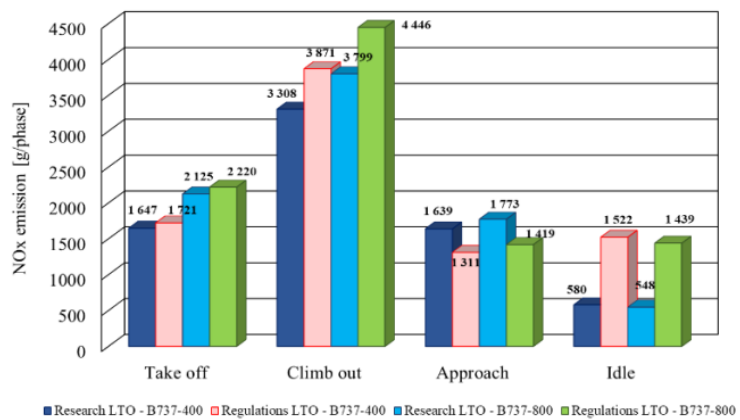

Fig. 10. $\mathrm{NO}_{\mathrm{x}}$ emission for $\mathrm{B} 737-400$ and $\mathrm{B} 737-800$ in regulations and adopted LTO cycle in each phase

In the case of emissions of nitrogen oxides, the results are not as diverse as in the case of hydrocarbons and carbon monoxide. Diversification concerns not only the value of emissions between particular phases, but also the emission of individual fumes exhaust gases in a given phase. The high $\mathrm{NO}_{\mathrm{x}}$ emission value can also be seen in the take-off phase, it is also caused by the high-power demand during the start operation. Figure 11 performed the total emission of individual toxic exhaust components in a standard LTO test and in the adopted LTO. It can be seen, that in the case of hydrocarbons, the difference in total emissions is as high as $60 \%$, while for carbon monoxide, $57 \%$. The smallest difference between the total emissions in calculated LTO tests is for $\mathrm{NO}_{\mathrm{x}}$ and it is $15 \%$. The difference is mainly due to the changes in times accepted in the adopted LTO, which was prepared especially for Poznań-Lawica Airport. It can also be said that the largest $\mathrm{CO}$ emission occurs during the entire test.

Another issue discussed here is the total emission of toxic exhaust components in both LTO tests for the B737800 aircraft (Fig. 12).

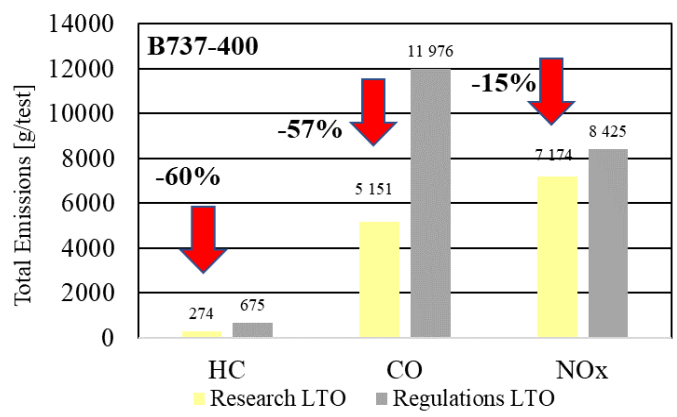

Fig. 11. Total emission of toxic exhaust fumes for adopted and regulations LTO for B737-400

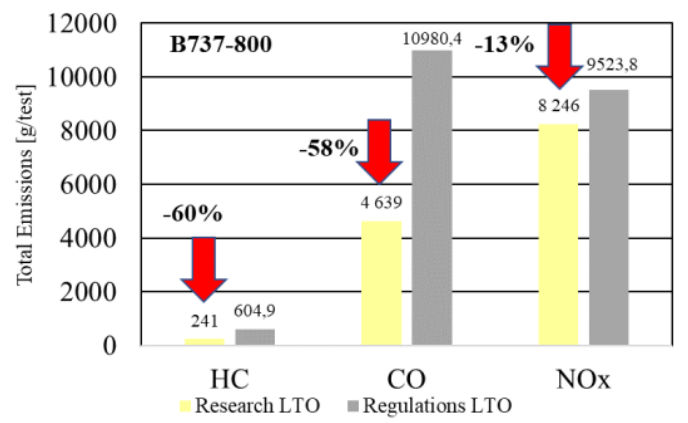

Fig. 12. Total emission of toxic exhaust fumes for adopted and regulations LTO for B737-800

The differences between the two tests are similar to those in the previous case. For the B737-800 aircraft, the total hydrocarbon emission in the LTO test was $60 \%$ lower, while in the case of carbon monoxide it was $58 \%$. The smallest difference occurs with the total emission of nitrogen oxides and amounts to $15 \%$. Emissions of individual toxic exhaust components are in the case of B737-400 and B737-800. In the case of the younger model - B737-800, the producers managed to reduce $\mathrm{HC}$ and $\mathrm{CO}$ emissions to a small extent, while the $\mathrm{NO}_{\mathrm{x}}$ emission increased, which most probably results from the highest power of engines fitted on the B737-400 model, and what is associated with it, the higher temperatures prevailing in the engine. 


\subsection{Analysis for Airbus A320 and A321}

The results of the calculations are presented below, in the form of graphs for Airbus A320 and A321 aircraft (Fig. 12). In the case of a hydrocarbon emission characteristic, the highest $\mathrm{HC}$ emission occurs in the Idle phase of the LTO test. Analyzing the graph, it can be seen that the higher emission is in the A320 model. In addition, the emission of hydrocarbons in the idle phase for A320 is more than $5000 \mathrm{~g} /$ phase higher with the standard LTO cycle than in the case of the adopted LTO. Analyzing the HC emission in the remaining phases of the test, it should be noted that the emission from the A320 model is in any case higher. The smallest hydrocarbon emission occurs in the Take-off phase of the LTO cycle. The emission volume, in each phase, between individual LTO tests is significant for the Airbus A320 model, while in the case of A321 (except the Idle phase) the difference in results are insignificant.

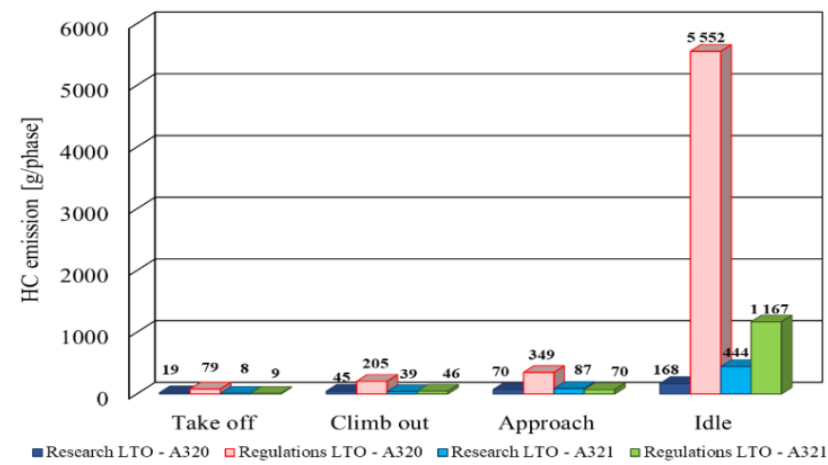

Fig. 12. HC emission for Airbus A320 and A321 in regulations and adopted LTO cycle in each phase

Figure 13 shows carbon monoxide emissions in each phase of both LTO test for Airbus A320 and A321. As in the previous case, the highest emission occurs in the Idle phase, however, it concerns the model of A321, not A320, where the emission of carbon monoxide is the highest in the Climb out phase. It should also be noted that the emissions in the climb out phase, in the case of the A320 model, is almost 30 times higher in the calculations carried out in accordance with the standard LTO test than in the adopted. In addition, there is a large difference in the $\mathrm{CO}$ emissions for the A320 model between the standard LTO cycle and the adopted LTO cycle. The next discussed characteristic (Fig. 14) is the emission of nitrogen oxides in the individual phases of the standard and test LTO test for the Airbus A320 and A321 models. The results presented in the chart are similar to those obtained in the Boeing B737-400 and B737-800 analysis. The largest amount of $\mathrm{NO}_{\mathrm{x}}$ is produced by Airbus A321 in the Climb out phase.

This is the phase during which the most nitrogen oxides are generated. The volume of emissions also reaches high values in the remaining test phase. While the $\mathrm{HC}$ emission was small, apart from the Idle phases, the $\mathrm{NO}_{\mathrm{x}}$ characteristic makes it clear that in each phase the emission is significant. The main reason for such a large emission of nitrogen oxides in the Take-off and Climb out phases is the high temperature in the engine during these phases. The high temperature is caused by the amount of power that must be used during these two phases.

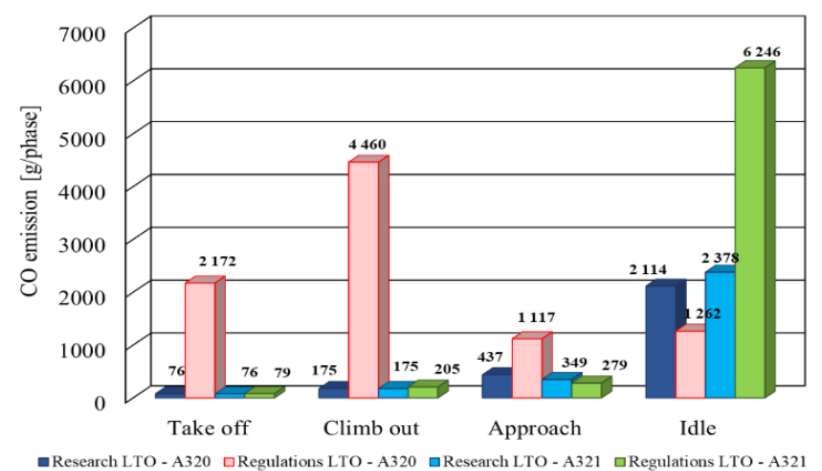

Fig. 13. CO emission for Airbus A320 and A321 in regulations and adopted LTO cycle in each phase

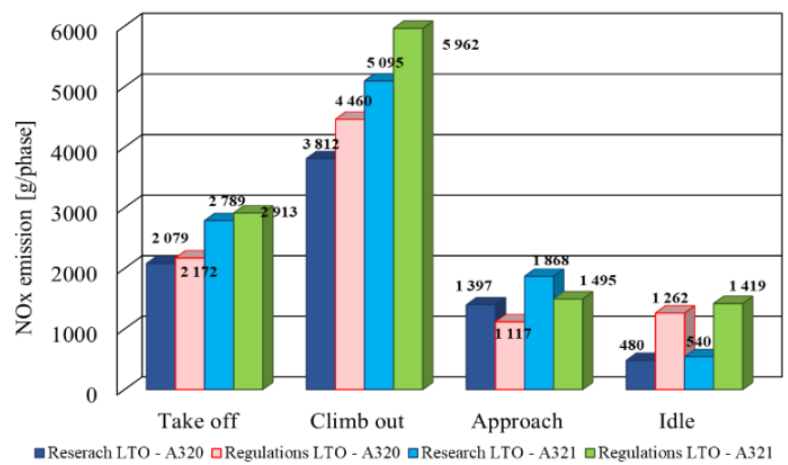

Fig. 14. $\mathrm{NO}_{\mathrm{x}}$ emission for Airbus A320 and A321 in regulations and adopted LTO cycle in each phase

At Figure 15 presented the total emission of toxic exhaust components in the standard and the LTO test for the Airbus A320. It can be seen, that in the case of hydrocarbons, the difference in total emissions is not as high as for Boeing, because reach about $47 \%$. For carbon monoxide the differences are about $55 \%$, which means almost the same as in Boeing emissions. The smallest difference between the total emission in calculated LTO tests is for $\mathrm{NO}_{\mathrm{x}}$ and it is $14 \%$. The difference is mainly due to the changes in times adopted in the LTO test, which was prepare especially for Poznań-Ławica Airport. It can also be said that the largest $\mathrm{NO}_{\mathrm{x}}$ emission occurs during the entire test. Another issue discussed is the total emission of toxic exhaust components in both LTO tests for the A321 aircraft (Fig. 16). The differences between the two tests are similar to those in the previous case.

For the A321 aircraft, the total hydrocarbon emission in the LTO test was $54 \%$ lower, while in the case of carbon monoxide it was $56 \%$. These results are similar to results for Boeing analysis. The smallest difference occurs with the total emission of nitrogen oxides and amounts to $13 \%$.

It can be seen, that in the case of the old model - Airbus A320 emission of exhaust gas fumes are smaller than for model A321. It can be cause for the size of models. Airbus A320 is larger and heavier than A320 which can entail bigger emission of exhaust gas fumes. 


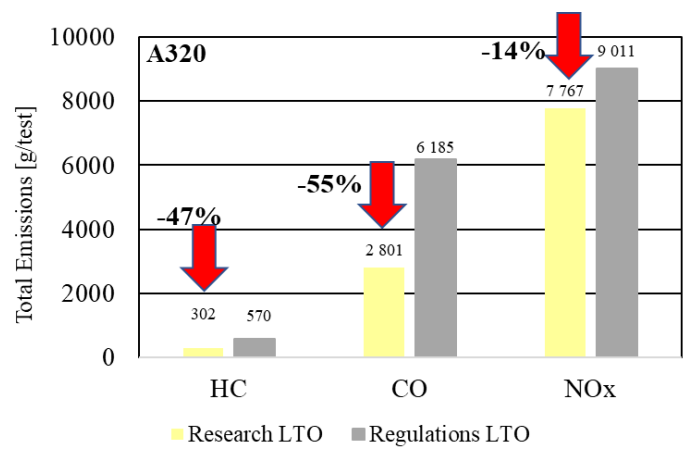

Fig. 15. Total emission of toxic exhaust fumes for adopted and regulations LTO for A320

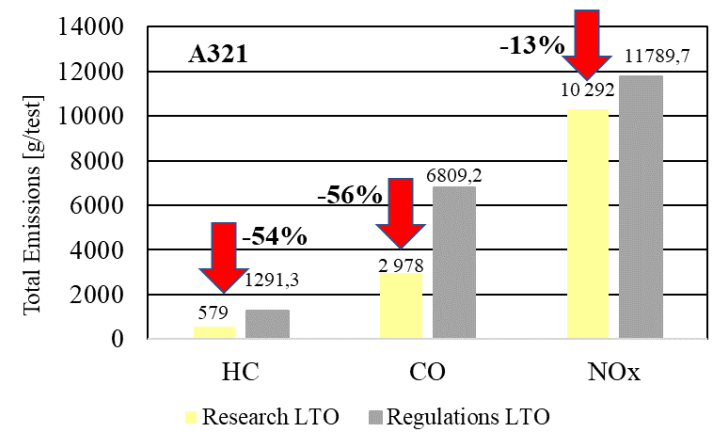

Fig. 16. Total emission of toxic exhaust fumes for adopted and regulations LTO for A321

\section{Conclusions}

Analysing the characteristics, it should be noted that the change in the time of individual phases of the LTO cycle has a significant impact on the volume of emissions from particular types of aircraft. For $\mathrm{HC}$ and $\mathrm{CO}$, emissions are reduced by $60 \%$. Analyzing the $\mathrm{NO}_{\mathrm{x}}$ emission, the differences are smaller, which is caused by the highest emission of this component during take-off, and in this phase the duration time was slightly reduced. The adopted confirms the validity of the thesis that parameters of flight phases adopted in the LTO test performed for the purpose of testing emissions in airport areas are not equal. For this type of adopted, individual parameters should be special for each airport to get results much closer to the actual local emission. The results confirm the suitability of adapting the LTO test to the airport conditions, in order to more thoroughly research and analyze the emissions of toxic gas compounds in the area of airports. Despite the choice of different aircraft models, the results are close to each other. Boeing aircrafts emit more $\mathrm{CO}$ than Airbus aircrafts, while the latter emit much more $\mathrm{NO}_{\mathrm{x}}$. On this basis, it is impossible to clearly determine which of the models is less conducive to polluting the environment, because each of them has its advantages and disadvantages. Significant differences occur in the emissions of adopted LTO. The presented methods performed that the emission in the case of Poznań-Ławica Airport is actually smaller than that obtained in the standard LTO test. It should be made aware of the appropriateness of adapting the LTO test to the real conditions prevailing at airports, in order to obtain more real results. There are significant differences in the case of the Poznań-Ławica airport, which is a medium-sized airport. It should be consider what impact the LTO cycle adjustment will have on results in the case of airports like London Heathrow, Paris - CDG, Amsterdam - Schiphol, Frankfurt Rhein-Main Airport or Barcelona-El Prat. This area is a field for future considerations.

\section{Bibliography}

[1] MERKISZ, J., PIELECHA, J., MARKOWSKI, J. et al. The analysis of air transport in Poland. International Conference on Air Transport INAIR 2015, 12-13 November, Amsterdam.

[2] Airbus 2016 Global Market Forecast.

[3] YIM, S.H.L., LEE, G.L., LEE, I.H. et al. Global, regional and local health impacts of civil aviation emissions. Environmental Research Letters. 2015, 10, 034001.

[4] Annex 16. Environmental protection. Vol. I: Aircraft noise. 2017, 4th ed. ICAO.

[5] NOWAK, M., JASIŃSKI, R., GALANT, M. Implementation of the LTO cycle in flight conditions using FNPT II MCC simulator. Material Science and Engineering. 2018, 421.

[6] Aircraft Engine Emissions Databank (2018), ICAO.

[7] ICAO environmental report. 2016 aviation and climate change.

Marta Galant, DEng. - Faculty of Transport Engineering, Poznan University of Technology.

e-mail:marta.galant@put.poznan.pl
[8] Manual Aircraft Instruction for Boeing B737-400

[9] Manual Aircraft Instruction for Boeing B737-800

[10] Manual Aircraft Instruction for Airbus A320.

[11] Manual Aircraft Instruction for Airbus A321.

[12] Cargo Boeing's 737 NG. Aviation International. 2016, 3, 11

[13] http://www.airline-empires.com/index.php?/gallery/image/ 7076-boeing-737-400-house/

[14] https://airleasecorp.com/aircraft/boeing/737_800

[15] https://fsxaibureau.com/manufacturing/airbus/airbus-a320/

[16] https://www.airbus.com/aircraft/passenger-aircraft/a320family/a321ceo.html

[17] GALANT, M., NOWAK, M., KARDACH, M. et al. Using the simulation technique to improve efficiency in General Aviation. AIP Conference Proceedings. 2019. 2078, 020097.

Marta Maciejewska, MEng. - Faculty of Transport Engineering, Poznan University of Technology.

e-mail:

marta.r.maciejewska@doctorate.put.poznan.pl

Prof. Paweł Fuc, DSc., DEng. - Faculty of Transport Engineering, Poznan University of Technology. e-mail:pawel.fuc@put.poznan.pl 